\title{
Clinicopathological significance of TM4SF5 expression in human hepatocellular carcinoma tissues
}

\author{
BAOJIN XU ${ }^{1}$, WU LV ${ }^{1}$, XIAOYAN LI ${ }^{2}$, LINA ZHANG ${ }^{2}$ and JIE LIN ${ }^{1}$ \\ Departments of ${ }^{1}$ General Surgery and ${ }^{2}$ Pathology, Cancer Hospital of China Medical University, \\ Liaoning Cancer Hospital and Institute, Shenyang, Liaoning 110042, P.R. China
}

Received October 1, 2018; Accepted February 22, 2019

DOI: $10.3892 /$ ol.2019.10210

\begin{abstract}
Hepatocellular carcinoma (HCC) is a major cause of cancer-related mortality. Recent studies have indicated that transmembrane $4 \mathrm{~L}$ six family member 5 (TM4SF5) serves a vital role in tumor progression and metastasis in human cell lines and mouse models. However, little is known about the association between TM4SF5 expression and clinicopathological factors. The aim of the current study was to investigate this association and evaluate overall survival (OS) of patients. Immunohistochemistry and clinical record analysis revealed that TM4SF5 expression was significantly downregulated in HCC tissues. In addition, TM4SF5 expression was significantly associated with tumor size, vascular invasion, tumor differentiation, and tumor-node-metastasis stage. The survival analysis also demonstrated that low TM4SF5 expression resulted in shorter OS. In conclusion, the association between TM4SF5 expression and clinicopathologic factors was established, and prognostic significance of TM4SF5 as a potential biomarker was evaluated using human HCC formalin-fixed paraffin-embedded tissue samples. The results of the present study demonstrated that low TM4SF5 expression was associated with tumor malignant progression and may be a good prognostic biomarker for OS in HCC.
\end{abstract}

\section{Introduction}

Hepatocellular carcinoma (HCC) is the fourth most common cancer and ranks third in digestive system in annual mortality worldwide (1). The early stage of the disease is characterized by dysplastic nodules, also known as preneoplastic lesions, which frequently develop in chronic inflammatory liver disease or hepatitis, and promote fibrosis, cirrhosis,

Correspondence to: Dr Jie Lin, Department of General Surgery, Cancer Hospital of China Medical University, Liaoning Cancer Hospital and Institute, 44 Xiaoheyan Road, Shenyang, Liaoning 110042, P.R. China

E-mail: lin_700410@163.com

Key words: transmembrane $4 \mathrm{~L}$ six family member 5, hepatocellular carcinoma, malignant progression, overall survival and HCC progression (2). In the past, surgery was the main treatment for HCC. To date, the prognosis of patients with HCC has improved due to various combinations of treatment strategies including, surgery, chemotherapy, and molecular-targeted therapy; however, therapeutic options remain limited.

Transmembrane $4 \mathrm{~L}$ six family member 5 (TM4SF5) is a transmembrane glycoprotein of the transmembrane $4 \mathrm{~L}$ six family, a branch of the tetraspanin family. The TM4SF5 gene is located on human chromosome 17q13.3, and encodes a 197 amino acid protein that has two cysteine residues and two N-glycosylation sites within the extracellular loops (3-5). TM4SF5 is highly expressed in esophageal cancer (6), colorectal cancer (7), and HCC cells (8). In the study of HCC, TM4SF5 has only been reported to induce HCC metastasis in cell line experiments (8). Therefore, there has been limited research on the association between TM4SF5 expression and clinicopathological factors in HCC.

In the present study, the clinicopathological significance of TM4SF5 expression was examined using formalin-fixed paraffin-embedded (FFPE) specimens from patients with HCC; the aim was to investigate the potential of TM4SF5 as a biomarker and to analyze the prognostic significance of TM4SF5 status.

\section{Materials and methods}

Patient selection. In this retrospective study, the pathology archives from the Liaoning Cancer Hospital and Institute (Shenyang, China) were reviewed to identify patients diagnosed with HCC. A spectrum of carcinoma cases was selected from the period between January 2009 and December 2011. Patients with intrahepatic cholangiocarcinoma or mixed hepatocholangiocarcinoma were not included in the present study; as a result, 89 cases of HCC were retrieved.

Clinical details were obtained from patients' pathology reports and original electronic medical records. The morphological features and histopathological variants were classified according to the National Comprehensive Cancer Network (NCCN) Clinical Practice Guidelines in Oncology (version 2018). In addition, other clinicopathological data, including age, sex, hepatitis B virus (HBV) or hepatitis $\mathrm{C}$ virus $(\mathrm{HCV})$ infection and survival data were obtained from medical records. Time interval between surgery and final follow-up 
Table I. Association between TM4SF5 expression and clinicopathological factors.

\begin{tabular}{|c|c|c|c|c|}
\hline \multirow[b]{2}{*}{ Clinicopathological factor } & \multirow[b]{2}{*}{ Number $(n=89)$} & \multicolumn{2}{|c|}{ TM4SF5 expression } & \multirow[b]{2}{*}{ P-value } \\
\hline & & Low & High & \\
\hline Sex & & & & 0.166 \\
\hline Male & 72 & 29 & 43 & \\
\hline Female & 17 & 10 & 7 & \\
\hline Age & & & & 0.650 \\
\hline$\leq 60$ & 64 & 29 & 35 & \\
\hline$>60$ & 25 & 10 & 15 & \\
\hline HBV infection & & & & 0.454 \\
\hline Negative & 9 & 5 & 4 & \\
\hline Positive & 80 & 34 & 46 & \\
\hline $\mathrm{HCV}$ infection & & & & 0.305 \\
\hline Negative & 76 & 35 & 41 & \\
\hline Positive & 13 & 4 & 9 & \\
\hline Tumor size $(\mathrm{cm})$ & & & & $<0.001$ \\
\hline$\leq 5$ & 59 & 11 & 48 & \\
\hline$>5$ & 30 & 28 & 2 & \\
\hline Vascular invasion & & & & $<0.001$ \\
\hline No & 75 & 26 & 49 & \\
\hline Yes & 14 & 13 & 1 & \\
\hline Tumor differentiation & & & & 0.006 \\
\hline G1 & 14 & 2 & 12 & \\
\hline G2 & 70 & 33 & 37 & \\
\hline G3 & 4 & 4 & 0 & \\
\hline TNM stage & & & & 0.005 \\
\hline IA & 12 & 1 & 11 & \\
\hline IB & 41 & 17 & 24 & \\
\hline II & 11 & 4 & 7 & \\
\hline IIIA & 10 & 5 & 5 & \\
\hline IIIB & 11 & 10 & 1 & \\
\hline IVB & 4 & 2 & 2 & \\
\hline
\end{tabular}

HBV, hepatitis B virus; HPC, hepatitis C virus; TM4SF5, transmembrane 4 L six family member 5; TNM, tumor-node-metastasis.

was identified as a patient's survival time. All aspects of this study were approved by the Liaoning Cancer Hospital and Institute Ethics Review Board (approval no. 20180902).

Tissue sections. Liver tissue sections were obtained from original FFPE tissue blocks. All tissue blocks were cut into regular $5-\mu \mathrm{m}$ sections and subsequently stained with hematoxylin for $3 \mathrm{~min}$ and eosin for $30 \mathrm{sec}$ (H\&E) at room temperature to confirm previously rendered histologic diagnoses based upon current World Health Organization classifications. In addition, all the pathologic slides were reviewed independently by two pathologists, who reevaluated tumor differentiation, lymph node metastasis, and vascular invasion.

Immunohistochemistry. Tissue samples from FPPE blocks were sectioned $(4 \mu \mathrm{m})$ and subsequently deparaffinized at $70^{\circ} \mathrm{C}$ for $120 \mathrm{~min}$, followed by antigen retrieval in citrate buffer, $\mathrm{pH}$ 6. The slides were incubated with 1:200 rabbit polyclonal anti-TM4SF5 primary antibody (cat. no. HPA041259; Sigma-Aldrich; Merck KGaA, Darmstadt, Germany). Subsequently, the sections were incubated by using UltraSensitiveTM SP (Mouse/Rabbit) IHC kit (cat. no. KIT-9710; Fuzhou Maixin Biotech Co., Ltd., Fuzhou, China) and expression was visualized using a DAB Detection kit (cat. no. DAB-0031; Fuzhou Maixin Biotech Co., Ltd.). High expression TM4SF5 positive control and untreated with primary antibody negative control were included.

Evaluations of the pattern of immunoreactivity, percentage of cell staining, and staining intensities for TM4SF5 were performed by two independent pathologists. The percentage of cells stained was graded on a scale of 1-4 (1, 1-25\%; 
Adjacent healthy tissue

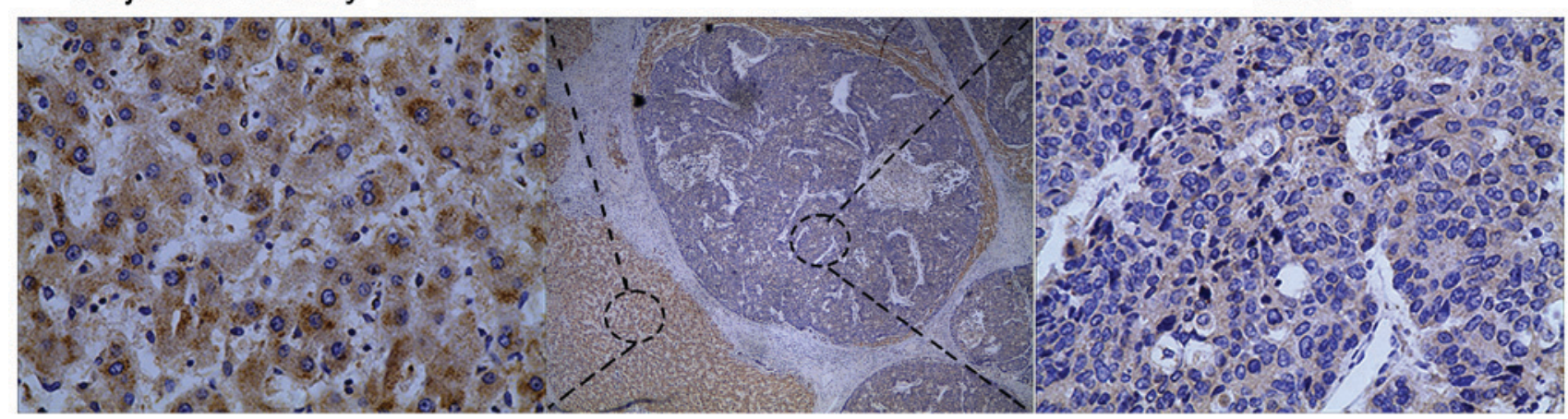

Figure 1. TM4SF5 expression in a liver tissue section. TM4SF5 protein expression is demonstrated by immunohistochemistry; $\mathrm{x} 40$ magnification (center image). The strong staining (score 8) in the adjacent healthy tissue (left) and the weak staining (score 3) in the tumor tissue (right) are presented in the photomicrographs; 4400 magnification. TM4SF5, transmembrane 4 L six family member 5.

$2,26-50 \% ; 3,51-75 \% ; 4,76-100 \%)$. Staining intensity was scored on a scale of 1-3 (1, weak; 2 , intermediate; 3 , strong). TM4SF5 expression was evaluated in each sample by calculating a final immunohistochemistry score as the sum of the percentage score and the intensity score. The tissue samples were divided into two groups, a high-TM4SF5 expression group with a total score $\geq 6$ and a low-TM4SF5 expression group with a total score $<6$.

Statistical analysis. Comparison of distribution among different histologies in this study was performed using a Wilcoxon signed-ranked test (GraphPad Prism software 7.0; GraphPad, La Jolla, CA, USA). $\chi^{2}$ test was used to assess the association between TM4SF5 expression and clinicopathologic parameters using SPSS 24.0 (SPSS, Inc., Chicago, IL, USA). The survival curves of the patients were determined using the Kaplan-Meier method and the log-rank test. $\mathrm{P}<0.05$ was considered to indicate a statistically significant difference.

\section{Results}

Clinicopathological characteristics. The study included 89 patients with HCC (Table I): 72 male, 17 female; median age at diagnosis was 54 years (range 25-76 years). The number of patients infected with HBV was $80(89.9 \%)$, whereas the number of patients infected with HCV was 13 (14.6\%). According to NCCN Clinical Practice Guidelines in Oncology (version 2018), 14 tumors were classified as well differentiated, 70 as moderately differentiated, and 4 as poorly differentiated carcinomas. Twelve patients $(13.5 \%)$ were staged as IA, $41(46.1 \%)$ as IB, $11(12.4 \%)$ as II, $10(11.2 \%)$ as IIIA, $11(12.4 \%)$ as IIIB, and $4(4.5 \%)$ as IVB.

Association between TM4SF5 expression and clinicopathological factors. TM4SF5 expression was investigated in 89 HCC tissues and corresponding adjacent normal tissues. Higher TM4SF5 protein expression was observed in normal tissues compared with carcinoma tissues (Fig. 1). To demonstrate the difference in immunohistochemistry scores between the two groups, a Wilcoxon signed-rank test was applied, and the result revealed that the difference was

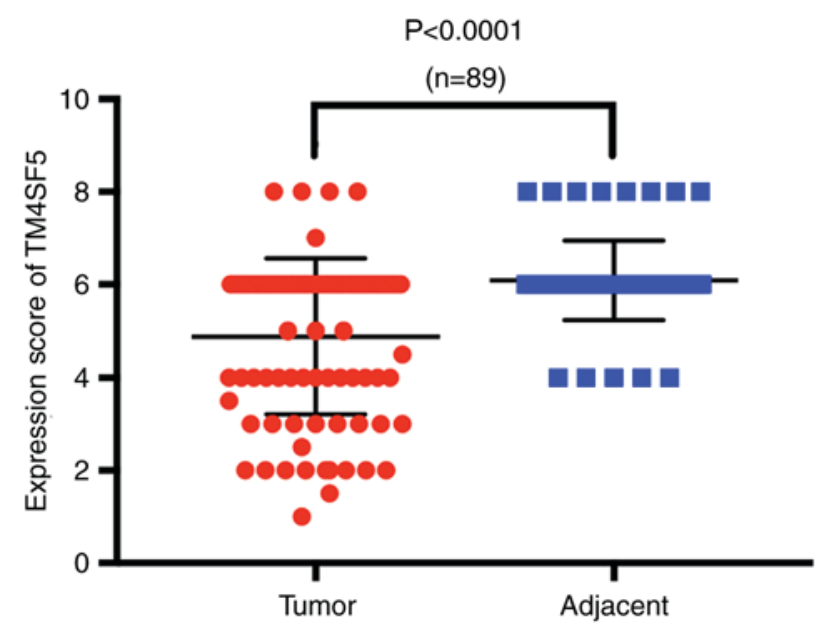

Figure 2. Scatter plot of TM4SF5 expression score differences between hepatocellular carcinoma tissues and adjacent normal tissues. TM4SF5, transmembrane $4 \mathrm{~L}$ six family member 5 .

statistically significant (Fig. 2). The carcinoma tissues were further divided into two groups; high-TM4SF5 expression and low-TM4SF5 expression (Fig. 3), to assess the association between TM4SF5 expression and clinicopathological factors. Tumor size, vascular invasion, tumor differentiation, and tumor-node-metastasis (TNM) stage were associated with low TM4SF5 expression (Table I). No significant associations were identified between TM4SF5 expression and the remaining factors.

Survival analysis. A total of 86 patients completed the follow-up period and the median period was 40 months (range 1-113 months). Among these, the number of patients in the high-TM4SF5 expression group was 47 and in the low-TM4SF5 expression group was 39. Kaplan-Meier survival analysis demonstrated that patients with low-TM4SF5 expression exhibited shorter overall survival (OS) compared with those with high-TM4SF5 expression, although the difference was not significant (Fig. 4A). In addition, the 5-year survival rate of the low-TM4SF5 expression group (30.8\%) was lower compared with the high-TM4SF5 expression group $(44.7 \%)$ (Fig. 4B). 

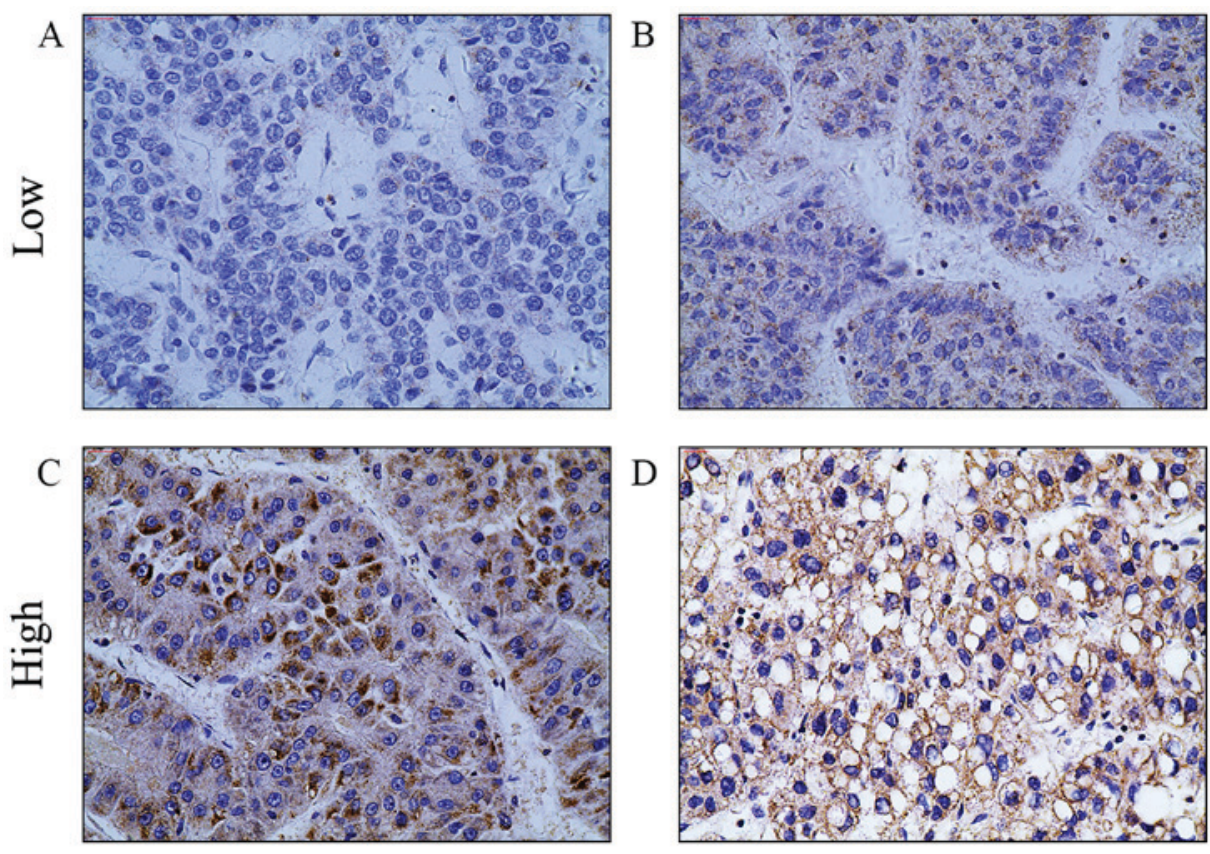

Figure 3. TM4SF5 protein expression in four hepatocellular carcinoma sections. (A and B) Weakly stained tissue. (C and D) Strong staining. Magnification, x400. TM4SF5, transmembrane $4 \mathrm{~L}$ six family member 5.
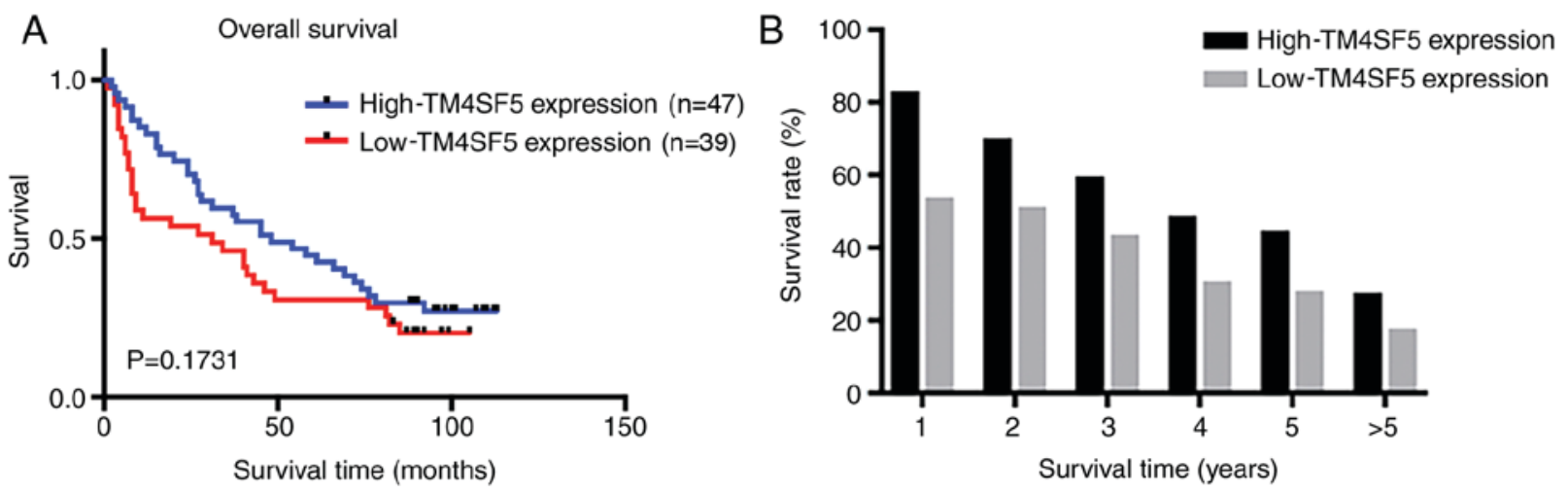

Figure 4. OS of patients with hepatocellular carcinoma. (A) Kaplan-Meier survival analysis of OS based on TM4SF5 expression score. (B) Bar graph of patient survival rates between high-TM4SF5-expression and low-TM4SF5-expression patient groups. OS, overall survival; TM4SF5, transmembrane 4 L six family member 5 .

\section{Discussion}

Human malignant tumors develop by genetic alterations and are made up of a heterogeneous population of cells. HCC develops through a multistep process, including cell mutation and regeneration; it is one of the most common cancers, and is characterized by a high mortality rate (9). Previous studies have demonstrated that the risk factors for the occurrence of HCC primarily include chronic HBV and HCV infections, chronic alcohol consumption, and non-alcoholic fatty liver disease (10-12). Despite the numerous therapeutic regimens for HCC that have been proposed, including etiology and oncology treatment, and currently approved standard therapies, the disease is still progressive for the majority of patients.

TM4SF5 is highly expressed in numerous types of cancers and serves a crucial role in tumorigenesis (6-8). In human hepatocytes, ectopic expression of TM4SF5 enhances focal adhesion kinase (FAK) Tyr ${ }^{577}$ phosphorylation as well as the association between FAK, Rho GTPase-activating protein 35 and Rho GTPase-activating protein 26, leading to transforming protein RhoA inactivation (13). Additionally, TM4SF5 induces stabilization of cytosolic cyclin dependent kinase inhibitor 1B (p27 ${ }^{\mathrm{kipl}}$ ), which may function as an inhibitor of the RhoA signaling pathway (14). Consequently, TM4SF5-mediated RhoA inactivation results in the process of epithelial-mesenchymal transition, leading to tumor cell migration, invasion, and proliferation due to the loss of contact inhibition (15). Tumor initiation and progression also involve complex communications between tumor cells and their microenvironments, including cytokines, extracellular matrix, and growth factors (16). In this complicated network, TM4SF5 protein not only cooperates with integrins to communicate with the microenvironment (17), but also mediates the activation of vascular endothelial growth factor transcription and secretion (18). This study suggests these factors contribute to the communication between cancer cells 
and extracellular environments, as well as angiogenesis, which provides beneficial conditions for tumor growth.

Advances in our knowledge about tumor initiation and progression have enriched the way the role of TM4SF5 is perceived. A study by Lee et al indicated that the interaction between TM4SF5 and CD44 was essential for the self-renewal and circulating capacities of HCC cells, leading to metastasis (19). In addition, mitogen-activated protein kinase 8 signaling activity has been demonstrated to regulate cell-cell adhesions through TM4SF5-mediated p $27^{\mathrm{kip} 1}$ phosphorylation (20). In the process of tumor immune escape, Ryu et al revealed that crosstalk between the TM4SF5/FAK pathway and the interleukin 6 (IL-6)/signal transducer and activator of transcription 3 pathway promoted metastatic potential by lowering IL- 6 expression levels and avoiding its immunological action (21). However, studies using human HCC tissues to evaluate the association between TM4SF5 expression and clinicopathological factors have been very limited.

In contrast with results of Lee et al (19), the present study demonstrated a cytoplasmic staining pattern for TM4SF5 in both tumors and corresponding adjacent normal cells. Notably, TM4SF5 expression was demonstrated to be significantly higher in adjacent normal cells compared with tumors. The data were separated into two groups, a low-TM4SF5 expression group and a high-expression group, to estimate the association between TM4SF5 expression and clinicopathological data. Low TM4SF5 expression was associated with increased tumor size, vascular invasion, tumor differentiation and TNM stage. In addition, survival analysis indicated that patients with low-TM4SF5 expression exhibited shorter OS, although no significance was identified. These results are contrary to previous findings, which may have resulted from the following: Experiments using cell lines and mice merely reflect the biological features of human bodies, whereas the initiation of human tumors is a complex, multistep process. The multistep development of human tumors involves several biological capabilities; for example, sustaining proliferative signaling, evading growth suppressors, resisting cell death, enabling replicative immortality, inducing angiogenesis, reprogramming of energy metabolism, evading immune destruction, and activating invasion and metastasis (22). By comparing the data of HCC microarray in GEO database (https://www.ncbi.nlm.nih.gov/gds/), it was found that the gene expression profiles and tumor biological functions were notably different due to individual discrepancy in the study of a series of patients with the same type of cancer. In the present study, the data of a number of patients with high-TM4SF5-expressing tumors were consistent with findings of a previous study (21). When all patient data were summarized, the results revealed distinct and opposing differences, which contributed to the conclusion that there are large differences in biological processes between cell lines, mice, and human bodies.

In the majority of previous studies $(5,18,19)$, a monoclonal antibody was applied to detect TM4SF5. By contrast, in the present study, only the commercial polyclonal antibody (HPA041259 from Sigma) was utilized; no comparison of immunohistochemistry specificity between two antibodies was performed, which may be a limitation to the present study.

In summary, associations between TM4SF5 expression and clinicopathological factors were identified, and the prognostic significance of TM4SF5 as a potential biomarker was further evaluated using a number of human HCC FFPE samples. To the best of our knowledge, this was the first study to evaluate TM4SF5 expression and clinicopathological factors using follow-up records and surgically resected specimens. The conclusion that high-TM4SF5 expression may be associated with OS was different from a previous study. These results indicated that TM4SF5 expression may not only be a prognostic factor, but also may be a predictive factor for HCC. However, a large-scale investigation is required to confirm these results.

\section{Acknowledgements}

The authors would like to thank Liaoning Cancer Hospital and Institute (Shenyang, China) for providing the tissue samples.

\section{Funding}

No funding was received.

\section{Availability of data and materials}

The datasets used and/or analyzed during the current study are available from the corresponding author upon reasonable request.

\section{Authors' contributions}

JL conceived and designed the study. BX and WL completed data extraction and analysis. XL and LZ performed the pathological evaluations. BX, XL and LZ drafted the manuscript. All authors read and approved the final manuscript.

\section{Ethics approval and consent to participate}

This study was approved by Liaoning Cancer Hospital and Institute Ethics Review Board (Shenyang, China) (approval no. 20180902). Written informed consent was obtained from the patients for use of their information and materials for research purposes.

\section{Patient consent for publication}

Not applicable.

\section{Competing interests}

The authors declare that they have no competing interests.

\section{References}

1. Siegel RL, Miller KD and Jemal A: Cancer statistics, 2019. CA Cancer J Clin 69: 7-34, 2019.

2. Tummala KS, Gomes AL, Yilmaz M, Graña O, Bakiri L, Ruppen I, Ximénez-Embún P, Sheshappanavar V, Rodriguez-Justo M, Pisano DG, et al: Inhibition of de novo NAD $(+)$ synthesis by oncogenic URI causes liver tumorigenesis through DNA damage. Cancer Cell 26: 826-839, 2014.

3. Lee SA, Park KH and Lee JW: Modulation of signaling between TM4SF5 and integrins in tumor microenvironment. Front Biosci (Landmark Ed) 16: 1752-1758, 2011.

4. Wright MD, Ni J and Rudy GB: The L6 membrane proteins-a new four-transmembrane superfamily. Protein Sci 9: 1594-1600, 2009. 
5. Lee SA, Ryu HW, Kim YM, Choi S, Lee MJ, Kwak TK, Kim HJ, Cho M, Park KH and Lee JW: Blockade of four-transmembrane L6 family member 5 (TM4SF5)-mediated tumorigenicity in hepatocytes by a synthetic chalcone derivative. Hepatology 49 : 1316-1325, 2009.

6. Wu YB, Huang YS, Xu YP, Sun YF, Yu DL, Zhang XQ, Long X, Zhu SQ, Zhou JL and Xu JJ: A high level of TM4SF5 is associated with human esophageal cancer progression and poor patient survival. Dig Dis Sci 58: 2623-2633, 2013.

7. Park BK, Park JY, Kim TH, Kim D, Wu G, Gautam A, Maharjan S, Lee SI, Lee Y, Kwon HJ and Choi KC: Production of an anti-TM4SF5 monoclonal antibody and its application in the detection of TM4SF5 as a possible marker of a poor prognosis in colorectal cancer. Int J Oncol 53: 275-285, 2018.

8. Lee D and Lee JW: Self-renewal and circulating capacities of metastatic hepatocarcinoma cells required for collaboration between TM4SF5 and CD44. BMB Rep 48: 127-128, 2015.

9. Amicone L and Marchetti A: Microenvironment and tumor cells: Two targets for new molecular therapies of hepatocellular carcinoma. Transl Gastroenterol Hepatol 3: 24, 2018.

10. Dutta R and Mahato RI: Recent advances in hepatocellular carcinoma therapy. Pharmacol Ther 173: 106-117, 2017.

11. Reeves HL, Zaki MY and Day CP: Hepatocellular carcinoma in obesity, type 2 diabetes, and NAFLD. Dig Dis Sci 61: 1234-1245, 2016.

12. Trojan J, Zangos S and Schnitzbauer AA: Diagnositics and treatment of hepatocellular carcinoma in 2016: Standrads and developments. Visc Med 32: 116-120, 2016.

13. Lee SA, Lee SY, Cho IH, Oh MA, Kang ES, Kim YB, Seo WD, Choi S, Nam JO, Tamamori-Adachi M, et al: Tetraspanin TM4SF5 mediates loss of contact inhibition through epithelialmesenchymal transition in human hepatocarcinoma. J Clin Invest 118: 1354-1366, 2008.
14. Besson A, Gurian-West M, Schmidt A, Hall A and Roberts JM: p27Kip1 modulates cell migration through the regulation of RhoA activation. Genes Dev 18: 862-876, 2004.

15. Kim H, Kang M, Lee SA, Kwak TK, Jung O, Lee HJ, Kim SH and Lee JW: TM4SF5 accelerates G1/S phase progression via cytosolic p27Kip1 expression and RhoA activity. Biochim Biophys Acta 1803: 975-982, 2010.

16. Hanahan D and Weinberg RA: The hallmarks of cancer. Cell 100: 57-70, 2000.

17. Sadej R, Romanska H, Baldwin G, Gkirtzimanaki K, Novitskaya V, Filer AD, Krcova Z, Kusinska R, Ehrmann J, Buckley CD, et al: CD151 regulates tumorigenesis by modulating the communication between tumor cells and endothelium. Mol Cancer Res 7: 787-798, 2009.

18. Choi S, Lee S-A, Kwak TK, Kim HJ, Lee MJ, Ye SK, Kim SH, Kim S and Lee JW: Cooperation between integrin alpha5 and teraspan TM4SF5 regulates VEGF-mediated angiogenic activity. Blood 113: 1845-1855, 2009.

19. Lee D, Na J, Ryu J, Kim HJ, Nam SH, Kang M, Jung JW, Lee MS, Song HE, Choi J, et al: Interaction of tetraspan(in) TM4SF5 with CD44 promotes self-renewal and circulating capacities of hepatocarcinoma cells. Hepatology 61: 1978-1997, 2015.

20. Kim H, Jung O, Kang M, Lee MS, Jeong D, Ryu J, Ko Y, Choi YJ and Lee JW: JNK signaling activity regulates cell-cell adhesions via TM4SF5-mediated p27(Kip1) phosphorylation. Cancer Lett 314: 198-205, 2012.

21. Ryu J, Kang M, Lee MS, Kim HJ, Nam SH, Song HE, Lee D and Lee JW: Cross talk between the TM4SF5/focal adhesion kinase and the interleukin-6/STAT3 pathways promotes immune escape of human liver cancer cells. Mol Cell Biol 34: 2946-2960, 2014

22. Hanahan D and Weinberg RA: Hallmarks of cancer: The next generation. Cell 144: 646-674, 2011. 